\title{
Cancer stem cells in hepatocellular carcinoma: recent progress and perspective
}

Tetsuhiro Chiba ${ }^{1,4}$, Akihide Kamiya ${ }^{2}$, Osamu Yokosuka $^{3}$, Atsushi Iwama ${ }^{1,4} *$

${ }^{1}$ Department of Cellular and Molecular Medicine, Graduate School of Medicine, Chiba University, 1-8-1 Inohana, Chuo-ku, Chiba 260-8670, Japan

${ }^{2}$ Division of Stem Cell Therapy, Center for Stem Cell and Regenerative Medicine, Institute of Medical Science, University of Tokyo, 4-6-1 Shirokanedai, Minato-ku, Tokyo 108-8639, Japan

${ }^{3}$ Department of Medicine and Clinical Oncology, Graduate School of Medicine, Chiba University, 1-8-1 Inohana, Chuo-ku, Chiba 260-8670, Japan

${ }^{4}$ JST, CREST, Sanbancho, Chiyoda-ku, Tokyo 102-0075, Japan

Key words; liver, stem cell, cancer stem cell, SP cell, hepatocellular carcinoma

Footnotes: *Corresponding author: Atsushi Iwama, M.D., PhD.

Department of Cellular and Molecular Medicine, Graduate School of Medicine, Chiba University, 1-8-1 Inohana, Chuo-ku, Chiba 260-8670, Japan

Telephone: $+81-43-2262189$

Fax: $+81-43-2262191$

E-mail: aiwama@faculty.chiba-u.jp 


\begin{abstract}
Although the "cancer stem cell (CSC)" hypothesis was first proposed roughly 50 years ago, recent progress in stem cell biology and technologies has successfully achieved the identification of CSCs in a variety of cancers. CSCs are defined as a minor population which possesses a prominent ability to generate new tumors that faithfully reproduce the phenotype of original tumors in xenotransplant assays. Additionally, CSCs are able to self-renew and generate differentiated progenies to organize a hierarchical cell system in a similar fashion to normal stem cells. Although not all types of cancer follow the CSC theory, it provides an attractive cellular mechanism to account for the therapeutic resistance and recurrence of the disease. A minor population with CSC properties has been detected in a number of established hepatocellular carcinoma (HCC) cell lines and extensive analyses characterizing the CSC system in primary HCC samples are now ongoing. Considering that HCC has high rates of recurrence and mortality, novel therapeutic approaches are urgently required. Although the clinical relevance of CSCs remains elusive, deep understanding of the cellular organization of HCC may allow us to develop therapies targeting specific cell types such as CSCs.
\end{abstract}




\section{Introduction}

Cancer is usually unicellular in origin $[1,2]$, although cancer cells constitute functional heterogeneity in a wide variety of cancers [3]. Classically, two general theories have been debated in terms of carcinogenesis [4]. The stochastic model indicates that a few cells which acquired proliferative potential via stochastic events are responsible for tumor formation. The alternative hypothesis, namely, the hierarchical model, postulates that a small subset of cells generates a hierarchical organization containing varied downstream descendants, proliferates extensively, and initiates tumors at high frequency.

Stem cells, generally defined by an ability to differentiate into multiple cell lineages and self-renew, contribute to not only organogenesis but also regeneration in response to the injury of tissues and organs [5]. Recent advancements in stem cell biology have allowed for the identification and characterization of stem cells in a variety of tissues and organs. On the other hand, it has been documented that solid tumors such as breast cancer and colon cancer contain a small subset of tumorigenic cells which can generate new tumors in xenograft transplantation $[6,7]$. This minor population of cells, termed cancer stem cells (CSCs) or tumor initiating cells (TICs), possesses stem cell-like properties and contributes to a hierarchical structure containing varied progenies in a similar fashion to normal stem cells. Successful detection of CSCs in a wide variety of cancers supports the hierarchical carcinogenesis theory.

Both normal stem cells and CSCs largely share surface marker phenotype and molecular machinery concerning self-renewal and differentiation. These phenotypic characteristics of CSCs have been well documented in hematological malignancies [8, 9], but little is shown in solid tumors including hepatocellular carcinoma (HCC). Furthermore, it is likely that CSCs are closely associated with not only carcinogenesis but also the recurrence and metastasis of tumors [10]. HCC is one of the most 
common malignancies worldwide and frequently shows strong resistance to traditional anticancer therapies such as chemotherapy and radiotherapy $[11,12]$. However, there is not sufficient evidence on CSCs in primary HCC. Thus, both the characterization and an understanding of the CSC system in liver are of paramount importance to elucidate mechanisms underlying hepatocarcinogenesis and to establish novel therapeutic approaches.

In this review, we will summarize the recent progress in CSC research in $\mathrm{HCC}$ and the molecular machinery underlying hepatocarcinogenesis. We also provide a perspective on therapeutic approaches against HCC from the CSC standpoint.

\section{Normal hepatic stem cells}

Normal adult hepatocytes are ordinarily in the quiescent $G_{0}$ state and mitotically inactive, although the liver regenerates quickly after acute injury and volume loss [13]. The regeneration is accomplished by the simple duplication of mature hepatocytes without the activation of stem/progenitor cells [14]. The presence of hepatic stem cells has been in doubt for some time. However, recent studies have successfully identified and characterized hepatic stem cells not only in fetal livers but also in adult livers (Table 1) $[15,16]$.

\subsection{Stem/progenitor cells in fetal livers}

In the developing liver, both fibroblast growth factor (FGF) and bone morphogenetic protein (BMP) signals promote the commitment of ventral endoderm to the liver bud (Figure 1) [17]. During liver bud growth, bipotent progenitors termed hepatoblasts proliferate and differentiate into hepatocytes and cholangiocytes under the control of various sets of transcription factors. Hepatoblasts could be identified as

RT1A $1^{-} \mathrm{OX} 18^{\text {low }} \mathrm{ICAM}_{-1}{ }^{+}, \quad \mathrm{Liv}^{+}, \quad$ E-cadherin $^{+}, \quad \mathrm{Dlk}^{+} \quad$ or




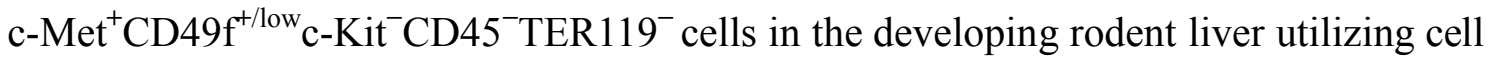
sorting technology and clonal colony assays [18-23]. Interestingly, a portion of

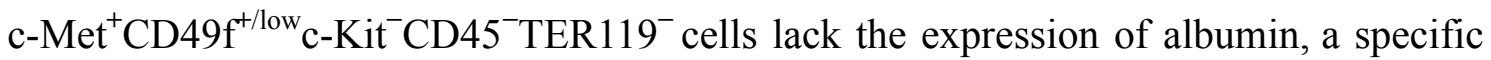
marker of hepatocyte differentiation. These albumin ${ }^{-}$cells give rise to albumin ${ }^{+}$ progenitor cells in response to hepatocyte growth factor (HGF) and differentiate into hepatocytes and cholangiocytes [24]. They also retain the potential to differentiate into non-hepatic lineages, pancreatic aciner cells and intestinal cells [23, 24]. These findings indicate that $\mathrm{c}-\mathrm{Met}^{+} \mathrm{CD} 49 \mathrm{f}^{+/ \text {low }} \mathrm{c}-\mathrm{Kit}^{-} \mathrm{CD} 45^{-}$TER $119^{-}$cells include a small number of pluripotent precursors of hepatoblasts or hepatic stem cells.

In an investigation of human fetal liver, Schmelzer et al. found that epithelial cell adhesion molecule $\left(\right.$ EpCAM/CD326) ${ }^{+}$cells can be divided into 2 subtypes [25]; (a) hepatoblasts positive for intercellular adhesion molecule-1 (ICAM-1), alpha-fetoprotein (AFP), albumin, cytokeratin 19 (CK19) and CD133, and (b) human hepatic stem cells (hHpSCs), accounting for less than $5 \%$ of $\mathrm{EpCAM}^{+}$cells, positive for CK19, CD133, neural cell adhesion molecule (NCAM) and claudin 3, but not for ICAM-1, AFP and albumin. These populations behaved differently in colony assays and hHpSCs are assumed to be pluripotent precursors of hepatoblasts (Figure 1).

\subsection{Stem/progenitor cells in adult livers and oval cells}

Although the identification of adult stem cells in normal liver has been a challenge for some time, recent studies have shown that $\mathrm{EpCAM}^{+}$liver cells purified from adult livers possess considerably similar biological characteristics to those from fetal livers and function as hepatic stem cells [25]. Given that some $\mathrm{EpCAM}^{+}$cells are also positive for CD133 and NCAM, it might be possible to further enrich the hepatic stem cell population. Prospective assays using these cells would be beneficial to promote investigations of both normal stem cells and CSCs in liver. 
Oval cells are defined as small cells with an oval nucleus and scanty cytoplasm and are considered to be progenitor cells with the ability to differentiate into hepatocytes and cholangiocytes $[26,27]$. They emerge from the periportal region in regenerating liver where the proliferation of mature hepatocytes is impaired. On the basis of phenotypic characteristics in common with hematopoietic stem cells (HSCs), such as the expression of cell surface markers, CD34, c-Kit, Sca-1, and Thy1, oval cells have been considered to originate from bone marrow [28, 29]. However, several reports have provided evidence that hepatic oval cells originate from the liver, not from bone marrow [30, 31]. Recently, Yovchev et al. reported that $\mathrm{EpCAM}^{+}$oval cells express not only epithelial markers such as AFP, CK19 and OV-1, but also mesenchymal markers such as vimentin, glypican1 and BMP7 [32]. These oval cells show decreased expression of CD34, c-Kit and NCAM. The origin of oval cells is still controversial. This could be partially due to the heterogeneity of oval cells.

Kuwahara et al. focused on the functional definition of stem/progenitor cells of hepatic origin using label-retaining cell (LRC) assays, widely utilized for the detection of somatic stem cells in many tissues and organs [33]. They conducted bromodeoxyuridine (BrdU) LRC assays in a mouse model of N-acetyl-p-aminophenol (APAP)-induced liver injury and successfully identified LRCs in the canal of Hering (proximal biliary tree), intralobular bile ducts, periductal mononuclear cells that lack hepatocytic and biliary markers, and peribiliary hepatocytes, suggesting that the liver has a multi-tiered, flexible system of regeneration rather than a single stem/progenitor cell location [33]. These findings clearly indicate the heterogeneity of hepatic stem/progenitor cells including oval cells and the need to clarify the extent to which each subset contributes to regeneration of the liver.

\section{Cancer stem cells in liver}


Dysregulated self-renewal capability, following oncogenic mutations, is one of the key events in the early stages of carcinogenesis [34]. It is believed that CSCs usually arise from normal stem/progenitor cells with enhanced or acquired self-renewal capability (Figure 2). However, it remains unclear whether this hypothesis can be applied to hepatocarcinogenesis. Many attempts have been made to detect a small subset of cancer cells with the characteristics of CSCs in HCC (Table 2).

Side population (SP) cell sorting is useful for detecting CSCs in various cancer cell lines [35]. The SP phenotype is determined by the ability to efflux the dye Hoechst 33342 through an adenosine triphosphate (ATP)-binding cassette (ABC) membrane transporter [36]. Only $0.25-0.80 \%$ of Huh7 and PLC/PRF/5 HCC cells exhibits the SP phenotype. Of note, tumor initiating capacity detected in xenotransplantation assays using immunodeficient mice has been strictly confined to SP cells [37]. One thousand SP cells was enough to generate tumors in xenotransplantation, while at least $1 \times 10^{6}$ unsorted $\mathrm{HCC}$ cells were required for tumor formation, suggesting that TICs are enriched by SP cell sorting at least 1,000-fold. Considering that the frequency of HCC SP cells was less than $1 \%$, the minority cell population detected as SP cells, but not non-SP cells, might possess tumorigenic potential in these HCC cells. However, several paradoxes have been reported to this technique. C6 glioma cells contain approximately $0.4 \%$ SP cells. In serum-free medium, C6 SP cells, but not non-SP cells are responsible for the in vivo tumorigenesis [38]. On the other hand, most C6 cells cultured in serum-containing medium, including both SP and non-SP cells exhibit tumor initiating capacity in vivo, raising the possibility that C6 cells do not follow cancer stem cell theory and that non-SP cells easily suffer Hoechst toxicity and fail to grow under serum-free conditions [39, 40]. The similar findings that exclude SP cell sorting technique from the major defining markers of cancer stem cells are also reported in other cancer cell types [41]. Thus, we should be more careful when applying SP cell 
sorting to cancer stem cell analysis including culture conditions and Hoechst cytotoxicity.

HCC cells positive for CD133, a potential cell surface marker for CSCs in a number of tumors, have been reported to exhibit greater tumorigenicity than the corresponding $\mathrm{CD}^{-} 33^{-}$cells in $\mathrm{HCC}$ cell lines $[42,43]$. In mouse models of $\mathrm{HCC}$, methionine adenosyltransferase I, alpha (Matla) knockout mice and Pten knockout mice, tumorigenic capacity was mainly detected in $\mathrm{CD}_{133^{+}}$oval cells $[44,45]$. OV6 and EpCAM are also reported as specific surface markers for CSCs in HCC cell lines [46, 47].

In contrast to HCC cell lines, HCC cells from surgical specimens hardly engrafted in immunodeficient mice. Recently, $\mathrm{CD} 90^{+} \mathrm{CD} 44^{+}$cells were reported to engraft in the livers of severe combined immunodeficient (SCID)/Beige mice and behave as CSCs [48]. However, the engraftment of these cells was very inefficient and no obvious tumor masses developed. To prove that CSCs exist in human primary HCC, technical improvements to obtain better engraftment in xenotransplantation are needed. The usage of more immunodeficient mice and longer observation periods would be the approach to try first as reported [49].

\section{Cell origin of liver malignancies}

The cell origin of $\mathrm{HCC}$ has long been debated, but whether $\mathrm{HCC}$ originates from mature hepatocytes or stem/progenitor cells remains unclear. HCC usually develops against a setting of chronic liver injury due to chronic infection of hepatitis viruses [11]. Most well-differentiated HCCs in the early stages are detected as a small lesion with a normal level of serum AFP. Subsequently, they increase in diameter and become moderately to poorly differentiated cancerous tissues producing AFP. These findings imply that HCC might develop and progress during the de-differentiation of mature hepatocytes. 
On the other hand, the concept of blocked ontogeny, that maturation arrest in stem/progenitor cells contributes to cancer development, is also accepted in hepatocarcinogenesis [50]. It is evident that transformed oval cells could be a cellular origin of liver tumors $[51,52]$. Additionally, the activation of oval cells has been observed in not only various rodent models of carcinogenesis but also in human chronic liver disease, HCC and CCC $[53,54]$. Combined hepatocellular and cholangiocellular carcinoma (HC-CC) is a rare but distinct type of liver malignancy. Histological analyses revealed the proliferation of an oval-cell-like subpopulation to varying degrees except in the HCC and CC components, which indicates the role of stem/progenitor cells as the cellular origin of the tumor [55]. Consistent with this, it has been also reported that fetal liver-derived hepatic stem/progenitor cells transduced with Bmil or mutant $\beta$-catenin acquired enhanced self-renewal capability and tumorigenicity to initiate HC-CC [56]. These observations imply that dysregulated propagation of hepatic stem/progenitor cells is an important early step in hepatocarcinogenesis. Similarly, the implantation of p53-null oval cells resulted in the development of HC-CC in recipient mice [57]. Therefore, HC-CC might be derived from hepatic stem/progenitor cells at least in some instances (Figure 2).

Interestingly, it was reported that HCC could be divided into distinct subtypes sharing gene expression features with subsets of cells in differing stages of differentiation and the HCC subtypes with a similar gene expression profile to hepatic stem/progenitor cells had a poor prognosis [58, 59]. Thus, HCC might develop from more heterogeneous cells during differentiation (Figure 2).

\section{Molecular machinery operating in normal and cancer stem cells in liver}

Cancer-related signaling pathways, such as the Wnt, Shh, Notch and PI3K/AKT/mTOR pathways, play an important role in the maintenance or augmentation of the 
self-renewal capability of CSCs as well as normal stem cells (Figure 3) [60, 61]. Interestingly, however, the dependency on these signals differs somewhat between normal stem cells and CSCs [62]. Understanding the molecular mechanisms operating in normal stem cells and CSCs is essential to innovate novel therapeutic approaches.

\subsection{Polycomb-group (PcG) gene products}

PcG proteins are transcriptional repressors that function by modulating chromatin structure. They form chromatin-associated multiprotein complexes, polycomb repressive complex (PRC) 1 and PRC2 [63]. Bmi1, one of the components of PRC1, regulates the cell cycle, apoptosis and senescence by repressing the Ink4a/Arf tumor suppressor gene locus [64]. Bmi1 is essential for the maintenance of the self-renewal capability of somatic stem cells including hepatic stem/progenitor cells [58]. On the other hand, overexpression of Bmil in hepatic stem/progenitor cells augments their self-renewal capability and induces tumor development in mice [58]. Consistent with these findings, BMI1 is overexpressed in a number of tumors [65]. Of note is that it is preferentially expressed in $\mathrm{CD}_{4} 4^{+} \mathrm{CSCs}$ in head and neck tumors [66] and in tumor-initiating SP cells in HCC cell lines [67]. Expression levels of BMI1 in HCC cell lines are faithfully correlated with the proportion of SP cell fraction and tumor-initiating capacity in mice [67]. Furthermore, levels of BMI1 expression in HCC were well correlated with the progression and prognosis of the disease [68]. These findings suggest that Bmil regulates self-renewal of both normal stem cells and CSCs by repressing the transcription of negative regulator genes for stem cell maintenance such as Ink4a and $\operatorname{Arf}$ [63] and by doing so, acts against oncogene-induced senescence, which is of substantial importance to the elimination of transforming cells that potentially develop into CSCs [69].

The important role of Ezh2, one of the components of PRC2, has been also 
recognized recently. EZH2 is also overexpressed in a variety of cancers including HCC [65]. Elucidation of its role in normal stem cells and CSCs requires further analysis.

\subsection{Wnt/ $\beta$-catenin signaling}

$\mathrm{Wnt} / \beta$-catenin signaling is a general regulator of self-renewal in a wide range of stem cell systems and closely associated with carcinogenesis [70]. It has been demonstrated that murine hepatic stem/progenitor cells transduced with mutant $\beta$-catenin acquired excessive self-renewal capability and tumorigenicity in a similar fashion to Bmil [58]. In addition, Yang et al. reported that $\mathrm{Wnt} / \beta$-catenin signaling is activated in both rodent oval cells and $\mathrm{OV}^{+}$tumorigenic $\mathrm{HCC}$ cells [46]. These findings indicate that $\mathrm{Wnt} / \beta$-catenin signaling is involved in the development and maintenance of CSCs.

Hepatoblastoma $(\mathrm{HB})$ is a pediatric liver tumor. Because it shows various morphological patterns including epithelial and mesenchymal lines of differentiation, HB is considered to be derived from developmentally primitive pluripotent stem cells in some instances. Aberrant activation of $\mathrm{Wnt} / \beta$-catenin signaling due to deletions or mutations of $\beta$-catenin, adenomatous polyposis coli (APC), and Axin is frequently observed in HBs [71]. It is reported that HBs could be divided into two subclasses, namely immature and differentiated subtypes, based on their genetic features [72]. Of interest, transcriptional programs driven by the activated Wnt/ $\beta$-catenin signaling differ considerably between the two subtypes, and additional Myc activation plays an important role in the conversion of differentiated tumors into immature ones. This highlights the important role of dysregulated Wnt/ $\beta$-catenin signaling in the transformation of stem/progenitor cells.

5.3. Transforming growth factor beta (TGF- $\beta$ ) signaling 
The TGF- $\beta /$ Smad signaling pathway is involved in the self-renewal and differentiation of stem cells and carcinogenesis in a variety of tissues and organs [73]. Tang et al. reported that hepatic stem/progenitor cells express TGF- $\beta$ signaling-related proteins, TGF- $\beta$ receptor type II (TBRII) and embryonic liver fodrin (ELF), in post-transplant human liver tissues [74]. In addition, they assumed that the activated IL-6/Stat3 pathway concomitant with the impaired TGF- $\beta$ signaling in these cells is relevant to the hepatocarcinogenesis in elf ${ }^{+-}$mice, which spontaneously develop HCC. This implicates the importance of TGF- $\beta$ and IL-6 signaling in the CSC population in HCC.

\section{Therapeutic resistance of cancer stem cells in liver}

Therapeutic resistance to standard chemotherapy and radiotherapy has been attributed to CSCs in a wide spectrum of cancers [75]. Many different types of cancer cells show overexpression of $\mathrm{ABC}$ transporters and drug resistance genes [76]. High drug efflux capacity through $\mathrm{ABC}$ transporters is one of the most striking characteristics of SP cells, a rare subset of CSCs in various cancer cell lines. Consistent with this, HCC SP cells were reported to exhibit resistance to anti-cancer agents such as doxorubicin [77]. In a human acute myelogenous leukemia (AML) xenotransplantation model, leukemic stem cells (LSCs) engrafted in the bone marrow niche, where they stayed in a quiescent $\mathrm{G}_{0}$ state. Surprisingly, up to $70 \%$ of CSCs survive cell cycle-dependent cytotoxic treatment, while LSC progenies are effectively eradicated [78]. Given the large population of HCC SP cells in $\mathrm{G}_{0}$ phase [79], it is conceivable that CSCs in HCC also show resistance to cell cycle-specific agents.

Bao et al reported that CSCs in glioma possess an efficient DNA repair system through the activation of Chk1 and Chk2 checkpoint kinases and show resistance to irradiation therapy [80]. Although the cyclin-dependent kinase inhibitor $\mathrm{p} 21^{\text {Waf1 }}$ could function as a tumor suppressor, a recent study showed that activation of $\mathrm{p} 21^{\text {Wafl }}$ is 
critical for DNA repair to maintain LSCs by preventing the accumulation of DNA-damage [81]. $\mathrm{p} 21^{\mathrm{WAF} 1}$ is preferentially expressed in HCC tissues rather than surrounding non-tumor tissues [82]. It is possible that well-developed DNA repair machinery operates in HCC CSCs and confers resistance to radiation therapy.

\section{Therapeutic approaches for liver cancer stem cells}

Although the investigation of treatments targeting CSCs in HCC has just started, strategies reported for CSCs of other tumors may offer hints for novel therapeutic approaches in HCC.

\subsection{Molecular target therapy}

The inhibition of CSC-specific pathways is one promising therapeutic approach. For example, LSCs of chronic myelogenous leukemia (CML) reside in the bone marrow niche in a quiescent $\mathrm{G}_{0}$ state and are resistant to chemotherapy and targeted therapies. Nuclear protein promyelocytic leukemia protein (PML) is essential for keeping LSCs in a quiescent state. Pharmacological inhibition of PML has been shown to change LSCs sensitive to conventional and targeted therapies by recruiting them into the cell cycle [83]. Ma et al. documented that activation of the Akt/PKB and Bcl-2 pathway contributes to the chemoresistance observed in $\mathrm{CD} 133^{+} \mathrm{HCC}$ cells [84]. It is noteworthy that treatment with an Akt1 inhibitor sensitized CD133 ${ }^{+} \mathrm{HCC}$ cells to conventional anti-cancer drugs such as 5-FU. Aldehyde dehydrogenase (ALDH), a detoxifying enzyme which eliminates toxic byproducts of reactive oxygen species (ROS), is a marker of both normal stem cells and CSCs. It was found that ALDH is highly expressed and confers chemoresistance to alkylating agents such as cyclophosphamide in LSCs and breast CSCs $[85,86]$. Given that the majority of $\mathrm{CD}_{133}{ }^{+}$cells in HCC cell lines also show strong ALDH enzymatic activity [87], ALDH 
inhibitors might be effective for the eradication of CSCs in HCC.

\subsection{Differentiation therapy}

It is presumed that the differentiation of CSCs ultimately results in the suppression of carcinogenesis, because the tumorigenicity of CSCs is largely determined by their own self-renewal capability. It has been documented that BMPs promote the differentiation of glioma stem cells and reduce their tumorigenic potential [88]. In transgenic mice in which the expression of c-Myc is conditionally regulatable, c-Myc expression induced multiple HCCs. Upon the inactivation of Myc, HCC cells lost neoplastic properties and differentiated into hepatocytes and cholangiocytes [89]. The mice showed a decrease in tumor volume and prolonged survival. Hepatocyte nuclear factor (HNF) $4 \alpha$ is a central transcription factor essential for hepatogenesis [90]. A recent report showed that the gene transfer of $H N F 4 \alpha$ reduced a population of tumorigenic $\mathrm{CD}^{+} 0^{+}$ and $\mathrm{CD}_{133^{+}}$cells purified from $\mathrm{HCC}$ cell lines by inducing differentiation of these subpopulations [91]. Interferon therapy is effective for not only eradicating the hepatitis viruses but also preventing the development of $\mathrm{HCC}$ regardless of the virological response. Interferon alpha treatment accelerated hepatocytic and biliary differentiation in oval cell lines [92]. Thus, interferon could be applied to the treatment of HCC for targeting CSCs.

\subsection{Antibody therapy}

Monoclonal antibody therapy is considered an important therapeutic modality for cancer. Rituximab (Anti-CD20) has already proven effective against lymphoid malignancies [93]. Although CD44, a receptor for hyaluronic acid and osteopontin, is widely expressed in both HSCs and LSCs, a monoclonal antibody specific to CD44 had a favorable effect on eradicating LSCs without affecting normal HSCs in a xenograft 
mouse model of human AML [94]. The administration of the CD44 antibody diminished the capacity of LSCs to home to the supportive microenvironment and promoted the terminal differentiation of LSCs in vivo. Given that CD44 ${ }^{+}$cells function as CSCs in a variety of solid tumors including HCC [6, 48], anti-CD44 antibody therapy might be a promising CSC-specific treatment in HCC.

Brain tumor stem cells reside in close proximity to blood vessels, called "vascular niches", where they receive signals that allow them to self-renew and to generate transit-amplifying cells [95]. Anti-angiogenic approaches such as the administration of anti-vascular endothelial growth factor (VEGF) monoclonal antibody disrupt vascular niches and dramatically reduce the number of CSCs. On losing the niches, CSCs cannot self-renew and differentiate into transit-amplifying cells. It is well-known that moderately to poorly differentiated HCCs are abundant in tumor vessels. Anti-angiogenic agents such as bevacizumab have already entered clinical trials for HCC and shown efficacy in some instances [96]. However, the CSC niche in HCC remains elusive. Identification of the microenvironment supportive for HCC progression is definitively needed.

\section{Perspective}

CSC research for HCC is somewhat behind that for other solid tumors. The major problem is that we are not sure of their existence in primary HCC. As described above, HCC cells from surgical specimens hardly engraft in conventional immunodeficient mice. This technical problem prevents us from obtaining an overall view of the CSC system in primary HCC. However, recent advances in xenotransplantation, including the co-injection of Matrigel (basement membrane matrix), usage of more immunodeficient nonobese diabetic / severe combined immunodeficiency (NOD/SCID) / interleukin-2 receptor (IL-2R) $\gamma^{\text {null }}$ (NOG) mice in xenotransplantation, and longer 
observation periods have considerably improved the engraftment of human cancer cells. By using these approaches, approximately $25 \%$ of unselected melanoma cells have been substantiated to possess tumorigenic potential [49]. Moreover, as few as 10 cells purified from murine lymphoma and AML were sufficient for the development of original hematological malignancies in syngenic transplantation assays [97]. These findings pointed out the possibility that the frequency of CSCs was underestimated in xenotransplant experiments because of a microenvironment unable to support the engraftment of donor cells. At the same time, these findings indicated that not all types of cancers fit a CSC model (Figure 4).

Does HCC follow the CSC theory? This is a very important question. Without answering it, we cannot solve other issues, including the origin of $\mathrm{HCC}$, niche for HCC, and mechanism of chemoresistance observed in HCC. Further efforts to identify and characterize HCC CSCs using improved xenotransplantation systems would provide a whole picture of the cellular organization of HCC. 


\section{Acknowledgement}

The preparation of this review was supported in part by the Chiba Serum Institute Memorial Fund for Health Medical Welfare and by grants for Global Center of Excellence Program from the Ministry of Education, Culture, Sports, Science and Technology, Japan, and Core Research for Evolutional Science and Technology (CREST) of Japan Science and Technology Corporation (JST).

\section{Conflicts of Interest Statement}

None Declared 


\section{References}

1. P.J. Fialkow, S.M. Gartler, A. Yoshida, Clonal origin of chronic myelocytic leukemia in man, Proc. Natl. Acad. Sci. USA 58 (1967) 1468-1471.

2. P.J. Fialkow, Use of genetic markers to study cellular origin and development of tumors in human females, Adv. Cancer Res. 15 (1972) 191-226.

3. G.H. Heppner, Tumor heterogeneity, Cancer Res. 44 (1984) 2259-2265.

4. T. Reya, S.J. Morrison, M.F. Clarke, I.L. Weissman, Stem cells, cancer, and cancer stem cells, Nature 414 (2001) 105-111.

5. D.A. Shafritz, M. Oertel, A. Menthena, D. Nierhoff, M.D. Dabeva, Liver stem cells and prospects for liver reconstitution by transplanted cells, Hepatology 43 (2006) S89-98.

6. M. Al-Hajj, M.S. Wicha, A. Benito-Hernandez, S.J. Morrison, M.F. Clarke, Prospective identification of tumorigenic breast cancer cells, Proc. Natl. Acad. Sci. USA 100 (2003) 3983-3988.

7. C.A. O'Brien, A Pollett, S Gallinger, J.E. Dick, A human colon cancer cell capable of initiating tumour growth in immunodeficient mice, Nature 445 (2007) 106-110.

8. C.H. Jamieson, I.L. Weissman, E. Passegué, Chronic versus acute myelogenous leukemia: A question of self-renewal, Cancer Cell 6 (2004) 531-533.

9. J.C. Wang, J.E. Dick, Cancer stem cells: lessons from leukemia, Trends Cell Biol. 15 (2005) 494-501.

10. C.T. Jordan, M.L. Guzman, M. Noble, Cancer stem cells, N. Engl. J. Med. 355 (2006) 1253-1261.

11. A.S. Befeler, A.M. Di Bisceglie, Hepatocellular carcinoma: diagnosis and treatment, Gastroenterology 122 (2002) 1609-1619.

12. W.Y. Lau, E.C. Lai, Hepatocellular carcinoma: current management and recent advances, Hepatobiliary Pancreat. Dis. Int. 7 (2008) 237-257. 
13. G.K. Michalopoulos, M.C. DeFrances, Liver regeneration, Science 276 (1997) $60-66$.

14. S. Sell, Heterogeneity and plasticity of hepatocyte lineage cells, Hepatology 33 (2001) 738-750.

15. M.H. Walkup, D.A. Gerber. Hepatic stem cells: In search of, Stem cells 24 (2006) 1833-1840.

16. L. Mishra, T. Banker, J. Murray, S. Byers, A. Thenappan, A.R. He, et al, Liver stem cells and hepatocellular carcinoma, Hepatology 49 (2009) 318-329.

17. K.S. Zaret, M. Grompe, Generation and regeneration of cells of the liver and pancreas, Science 322 (2008) 1490-1494.

18. H. Kubota, L.M. Reid, Clonogenic hepatoblasts, common precursors for hepatocytic and biliary lineages, are lacking classical major histocompatibility complex class I antigen, Proc. Natl. Acad. Sci. USA 97 (2000) 12132-12137.

19. T. Watanabe, K. Nakagawa, S. Ohata, D. Kitagawa, G. Nishitai, J. Seo, et al, SEK1/MKK4-mediated SAPK/JNK signaling participates in embryonic hepatoblast proliferation via a pathway different from NF-кB-induced anti-apoptosis, Dev. Biol. 250 (2002) 332-347.

20. M. Nitou, Y. Sugiyama, K. Ishikawa, N. Shiojiri, Purification of fetal mouse hepatoblasts by magnetic beads coated with monoclonal anti-e-cadherin antibodies and their in vitro culture, Exp. Cell Res. 279 (2002) 330-343.

21. N. Tanimizu, M. Nishikawa, H. Saito, T. Tsujimura, A. Miyajima, Isolation of hepatoblasts based on the expression of Dlk/Pref-1, J. Cell Sci. 116 (2003) 1775-1786.

22. M. Oertel, A. Menthena, Y.Q. Chen, B. Teisner, C.H. Jensen, D.A. Shafritz, Purification of fetal liver stem/progenitor cells containing all the repopulation potential for normal adult rat liver, Gastroenterology 134 (2008) 823-832. 
23. A. Suzuki, Y.W. Zheng, S. Kaneko, M. Onodera, K. Fukao, H. Nakauchi, et al, Clonal identification and characterization of self-renewing pluripotent stem cells in the developing liver, J. Cell Biol. 156 (2002) 173-184.

24. A. Suzuki, A. Iwama, H. Miyashita, H. Nakauchi, H. Taniguchi, Role for growth factors and extracellular matrix in controlling differentiation of prospectively isolated hepatic stem cells, Development 130 (2003) 2513-2524.

25. E. Schmelzer, L. Zhang, A. Bruce, E. Wauthier, J. Ludlow, H.L. Yao, et al, Human hepatic stem cells from fetal and postnatal donors, J. Exp. Med. 204 (2007) 1973-1987.

26. E. Farber, Similarities in the sequence of early histological changes induced in the liver of the rat by ethionine, 2-acetylamino-fluorene, and 3'-methyl-4-dimethylaminoazobenzene, Cancer Res. 16 (1956) 142-149.

27. C.B. Rountree, L. Barsky, S. Ge, J. Zhu, S. Senadheera, G.M. Crooks, A CD133-expressing murine liver oval cell population with bilineage potential, Stem Cells 25 (2007) 2419-2429.

28. B.E. Petersen, J.P. Goff, J.S. Greenberger, G.K. Michalopoulos, Hepatic oval cells express the hematopoietic stem cell marker Thy-1 in the rat, Hepatology 27 (1998) $433-445$.

29. B.E. Petersen, B. Grossbard, H. Hatch, L. Pi, J. Deng, E.W. Scott, Mouse A6-positive hepatic oval cells also express several hematopoietic stem cell markers, Hepatology 37 (2003) 632-640.

30. A. Menthena, N. Deb, M. Oertel, P.N. Grozdanov, J. Sandhu, S. Shah, et al, Bone marrow progenitors are not the source of expanding oval cells in injured liver, Stem Cells 22 (2004) 1049-1061.

31. X. Wang, M. Foster, M. Al-Dhalimy, E. Lagasse, M. Finegold, M. Grompe, The origin and liver repopulating capacity of murine oval cells, Proc. Natl. Acad. Sci. 
USA 100 (2003) 11881-11888.

32. M.I. Yovchev, P.N. Grozdanov, H. Zhou, H. Racherla, C. Guha, M.D. Dabeva. Identification of adult hepatic progenitor cells capable of repopulating injured rat liver, Hepatology 47 (2008) 636-647.

33. R. Kuwahara, A.V. Kofman, C.S. Landis, E.S. Swenson, E. Barendswaard, N.D. Theise, et al, The hepatic stem cell niche: Identification by label-retaining cell assay, Hepatology 47 (2008) 1994-2002.

34. M. Al-Hajj, M.F. Clarke, Self-renewal and solid tumor stem cells, Oncogene 23 (2004) 7274-7282.

35. C, Wu, B.A. Alman, Side population cells in human cancers, Cancer Lett. 268 (2008) $1-9$.

36. C. Hirschmann-Jax, A.E. Foster, G.G. Wulf, J.G. Nuchtern, T.W. Jax, U. Gobel, et al, A distinct "side population" of cells with high drug efflux capacity in human tumor cells, Proc. Natl. Acad. Sci. USA 101 (2004) 14228-14233.

37. T. Chiba, K. Kita, Y.W. Zheng, O. Yokosuka, H. Saisho, A. Iwama, et al, Side population purified from hepatocellular carcinoma cells harbors cancer stem cell-like properties, Hepatology 44 (2006) 240-251.

38. T. Kondo, T. Setoguchi, T. Taga, Persistence of a small subpopulation of cancer stem-like cells in the C6 glioma cell line, Proc. Natl. Acad. Sci. USA 101 (2004) 781-786.

39. X. Zheng, G. Shen, X. Yang, W. Liu, Most C6 cells are cancer stem cells: Evidence from clonal and population analyses, Cancer Res 67 (2007) 3691-3697.

40. N. Platet, J.F. Mayol, F. Berger, F. Hérodin, D. Wion, Fluctuation of the SP/non-SP phenotype in the C6 glioma cell line, FEBS Lett. 581 (2007) 1435-1440.

41. U.D. Lichtenauer, I. Shapiro, K. Geiger, M. Quinkler, M. Fassnacht, R. Nitschke, et al. Side population does not define stem cell-like cancer cells in the adrenocortical 
carcinoma cell line NCI h295R, Endocrinology 149 (2008) 1314-1322.

42. S. Yin, J. Li, C. Hu, X. Chen, M. Yao, M. Yan, et al, CD133 positive hepatocellular carcinoma cells possess high capacity for tumorigenicity, Int. J. Cancer 120 (2007) $1444-1450$.

43. S. Ma, K.W. Chan, L. Hu, T.K. Lee, J.Y. Wo, I.O. Ng, et al, Identification and characterization of tumorigenic liver cancer stem/progenitor cells, Gastroenterology 132 (2007) 2542-2556.

44. C.B. Rountree, S. Senadheera, J.M. Mato, G.M. Crooks, S.C. Lu, Expansion of liver cancer stem cells during aging in methionine adenosyltransferase 1A-deficient mice, Hepatology 47 (2008) 1288-1297.

45. C.B. Rountree, W. Ding, L. He, B. Stiles, Expansion of CD133 expressing liver cancer stem cells in liver specific PTEN deleted mice, Stem Cells 27 (2009) 290-299.

46. W. Yang, H.X. Yan, L. Chen, Q. Liu, Y.Q. He, L.X. Yu, et al, Wnt/beta-catenin signaling contributes to activation of normal and tumorigenic liver progenitor cells, Cancer Res. 68 (2008) 4287-4295.

47. T. Yamashita, J. Ji, A. Budhu, M. Forgues, M. Forgues, W. Yang, H.Y. Wang, et al, EpCAM-positive hepatocellular carcinoma cells are tumor-initiating cells with stem/progenitor cell features, Gastroenterology 136 (2009) 1012-1024.

48. Z.F. Yang, D.W. Ho, M.N. Ng, C.K. Lau, W.C. Yu, P. Ngai, et al, Significance of CD90+ cancer stem cells in human liver cancer, Cancer Cell 13 (2008) 153-166.

49. E. Quintana, M. Shackleton, M.S. Sabel, D.R. Fullen, T.M. Johnson, S.J. Morrison, et al, Efficient tumour formation by single human melanoma cells, Nature 456 (2008) 593-598.

50. V.R. Potter, Phenotypic diversity in experimental hepatomas: the concept of partially blocked ontogeny. The 10th Walter Hubert Lecture, Br. J. Cancer 38 
(1978) 1-23.

51. M.L. Dumble, E.J. Croager, G.C. Yeoh, E.A. Quail, Generation and characterization of p53 null transformed hepatic progenitor cells: oval cells give rise to hepatocellular carcinoma, Carcinogenesis 23 (2002) 435-445.

52. P. Steinberg, R. Steinbrecher, S. Radaeva, P. Schirmacher, H.P. Dienes, F. Oesch, et al, Oval cell lines OC/CDE 6 and OC/CDE 22 give rise to cholangio-cellular and undifferentiated carcinomas after transformation, Lab. Invest. 71 (1994) 700-709.

53. K.N. Lowes, B.A. Brennan, G.C. Yeoh, J.K. Olynyk, Oval cell numbers in human chronic liver diseases are directly related to disease severity, Am. J. Pathol. 154 (1999) 537-541.

54. T. Roskams, Liver stem cells and their implication in hepatocellular and cholangiocarcinoma, Oncogene 25 (2006) 3818-3822.

55. N.D. Theise, J.L. Yao, K. Harada, P. Hytiroglou, B. Portmann, S.N. Thung, et al, Hepatic 'stem cell' malignancies in adults: four cases, Histopathology 43 (2003) $263-271$.

56. T. Chiba, Y.W. Zheng, K. Kita, O. Yokosuka, H. Saisho, M. Onodera, et al, Enhanced selfrenewal capability in hepatic stem/progenitor cells drives cancer initiation, Gastroenterology 133 (2007) 937-950.

57. A. Suzuki, S. Sekiya, M. Onishi, N. Oshima, H. Kiyonari, H. Nakauchi, et al, Flow cytometric isolation and clonal identification of self-renewing bipotent hepatic progenitor cells in adult mouse liver, Hepatology 48 (2008) 1964-1978.

58. J.S. Lee, J. Heo, L. Libbrecht, I.S. Chu, P. Kaposi-Novak, D.F. Calvisi, et al, A novel prognostic subtype of human hepatocellular carcinoma derived from hepatic progenitor cells, Nat. Med. 12 (2006) 410-416.

59. T. Yamashita, M. Forgues, W. Wang, J.W. Kim, Q. Ye, H. Jia, et al, EpCAM and alpha-fetoprotein expression defines novel prognostic subtypes of hepatocellular 
carcinoma, Cancer Res. 68 (2008) 1451-1461.

60. R. Pardal, M.F. Clarke, S.J. Morrison, Applying the principles of stem-cell biology to cancer, Nat. Rev. Cancer 3 (2003) 895-902.

61. R. Hill, H. Wu, PTEN, stem cells, and cancer stem cells, J. Biol. Chem. 2008 Dec 30. [Epub ahead of print]

62. O.H. Yilmaz, R. Valdez, B.K. Theisen, W. Guo, D.O. Ferguson, H. Wu, et al, Pten dependence distinguishes haematopoietic stem cells from leukaemia-initiating cells, Nature 441 (2006) 475-482.

63. M.E. Valk-Lingbeek, S.W. Bruggeman, M. van Lohuizen, Stem cells and cancer; the polycomb connection, Cell 118 (2004) 409-418.

64. I.K. Park, S.J. Morrison, M.F. Clarke, Bmi1, stem cells, and senescence regulation, J. Clin. Invest. 113 (2004) 175-179.

65. A. Sparmann, M. van Lohuizen, Polycomb silencers control cell fate, development and cancer, Nat. Rev. Cancer 6 (2006) 846-856.

66. M.E. Prince, R. Sivanandan, A. Kaczorowski, G.T. Wolf, M.J. Kaplan, P. Dalerba, et al, Identification of a subpopulation of cells with cancer stem cell properties in head and neck squamous cell carcinoma, Proc. Natl. Acad. Sci. USA 104 (2007) 973-978.

67. T. Chiba, S. Miyagi, A. Saraya, R. Aoki, A. Seki, Y. Morita, et al, The polycomb gene product BMI1 contributes to the maintenance of tumor-initiating side population cells in hepatocellular carcinoma, Cancer Res. 68 (2008) 7742-7749.

68. M. Sasaki, H. Ikeda, K. Itatsu, J. Yamaguchi, S. Sawada, H. Minato, et al, The overexpression of polycomb group proteins Bmil and EZH2 is associated with the progression and aggressive biological behavior of hepatocellular carcinoma, Lab. Invest. 88 (2008) 873-882.

69. M. Collado, M.A. Blasco, M. Serrano, Cellular senescence in cancer and aging, 
Cell 130 (2007) 223-233.

70. T. Reya, H. Clevers, Wnt signalling in stem cells and cancer, Nature 434 (2005) 843-850.

71. Y. Wei, M. Fabre, S. Branchereau, F. Gauthier, G. Perilongo, M.A. Buendia, et al, Activation of beta-catenin in epithelial and mesenchymal hepatoblastomas, Oncogene 19 (2000) 498-504.

72. S. Cairo, C. Armengol, A. De Reyniès, Y. Wei, E. Thomas, C.A. Renard, et al, Hepatic stem-like phenotype and interplay of Wnt/beta-catenin and Myc signaling in aggressive childhood liver cancer, Cancer Cell 14 (2008) 471-484.

73. L. Mishra, K. Shetty, Y. Tang, A. Stuart, S.W. Byers, The role of TGF-beta and Wnt signaling in gastrointestinal stem cells and cancer, Oncogene 24 (2005) 5775-5789.

74. Y. Tang, K. Kitisin, W. Jogunoori, C. Li, C.X. Deng, S.C. Mueller, et al, Progenitor/stem cells give rise to liver cancer due to aberrant TGF-beta and IL-6 signaling, Proc. Natl. Acad. Sci. USA 105 (2008) 2445-2450.

75. J.E. Visvader, G.J. Lindeman, Cancer stem cells in solid tumours: accumulating evidence and unresolved questions, Nat. Rev. Cancer 8 (2008) 755-768.

76. C.F. Higgins, Multiple molecular mechanisms for multidrug resistance transporters, Nature 446 (2007) 749-757.

77. N. Haraguchi, T. Utsunomiya, Inoue H, F. Tanaka, K. Mimori, G.F. Barnard, et al, Characterization of a side population of cancer cells from human gastrointestinal system, Stem Cells 24 (2006) 506-513.

78. F. Ishikawa, S. Yoshida, Y. Saito, A. Hijikata, H. Kitamura, S. Tanaka, et al, Chemotherapy-resistant human AML stem cells home to and engraft within the bone-marrow endosteal region, Nat. Biotechnol. 25 (2007) 1315-1321.

79. Y. Kamohara, N. Haraguchi, K. Mimori, F. Tanaka, H. Inoue, M. Mori, et al, The search for cancer stem cells in hepatocellular carcinoma, Surgery 144 (2008) 144 
119-124.

80. S. Bao, Q. Wu, R.E. McLendon, Y. Hao, Q. Shi, A.B. Hjelmeland, et al, Glioma stem cells promote radioresistance by preferential activation of the DNA damage response, Nature 444 (2006) 756-760.

81. A. Viale, F. De Franco, A. Orleth, V. Cambiaghi, V. Giuliani, D. Bossi, et al, Cell-cycle restriction limits DNA damage and maintains self-renewal of leukaemia stem cells, Nature 457 (2009) 51-56.

82. L.F. Qin, I.O. Ng, S.T. Fan, M. Ng, p21/WAF1, p53 and PCNA expression and p53 mutation status in hepatocellular carcinoma, Int. J. Cancer 79 (1998) 424-428.

83. K. Ito, R. Bernardi, A. Morotti, S. Matsuoka, G. Saglio, Y. Ikeda, et al, PML targeting eradicates quiescent leukaemia-initiating cells, Nature 453 (2008) 1072-1078.

84. S. Ma, T.K. Lee, B.J. Zheng, K.W. Chan, X.Y. Guan, CD133+ HCC cancer stem cells confer chemoresistance by preferential expression of the Akt/PKB survival pathway, Oncogene 27 (2008) 1749-1758.

85. S. Ma, T.K. Lee, B.J. Zheng, K.W. Chan, X.Y. Guan, Characterization of cells with a high aldehyde dehydrogenase activity from cord blood and acute myeloid leukemia samples, Stem Cells 23 (2005) 752-760.

86. C. Ginestier, M.H. Hur, E. Charafe-Jauffret, F. Monville, J. Dutcher, M. Brown, et al, ALDH1 is a marker of normal and malignant human mammary stem cells and a predictor of poor clinical outcome, Cell Stem Cell 1 (2007) 555-567.

87. S. Ma, K.W. Chan, T.K. Lee, K.H. Tang, J.Y. Wo, B.J. Zheng, et al, Aldehyde dehydrogenase discriminates the CD133 liver cancer stem cell populations, Mol. Cancer Res. 6 (2008) 1146-1153.

88. S.G. Piccirillo, B.A. Reynolds, N. Zanetti, G. Lamorte, E. Binda, G. Broggi, et al, Bone morphogenetic proteins inhibit the tumorigenic potential of human brain 
tumour-initiating cells, Nature 444 (2006) 761-765.

89. C.M. Shachaf, A.M. Kopelman, C. Arvanitis, A. Karlsson, S. Beer, S. Mandl, et al, MYC inactivation uncovers pluripotent differentiation and tumour dormancy in hepatocellular cancer, Nature 431 (2004) 1112-1117.

90. F. Parviz, C. Matullo, W.D. Garrison, L. Savatski, J.W. Adamson, G. Ning, et al, Hepatocyte nuclear factor 4alpha controls the development of a hepatic epithelium and liver morphogenesis, Nat. Genet. 34 (2003) 292-296.

91. C. Yin, Y. Lin, X. Zhang, Y.X. Chen, X. Zeng, H.Y. Yue, et al, Differentiation therapy of hepatocellular carcinoma in mice with recombinant adenovirus carrying hepatocyte nuclear factor-4alpha gene, Hepatology. 48 (2008) 1528-1539.

92. R. Lim, B. Knight, K. Patel, J.G. McHutchison, G.C. Yeoh, J.K. Olynyk, Antiproliferative effects of interferon alpha on hepatic progenitor cells in vitro and in vivo, Hepatology 43 (2006) 1074-1083.

93. B.D. Cheson, J.P. Leonard, Monoclonal antibody therapy for B-cell non-Hodgkin's lymphoma, N. Engl. J. Med. 359 (2008) 613-626.

94. L. Jin, K.J. Hope, Q. Zhai, F. Smadja-Joffe, J.E. Dick, Targeting of CD44 eradicates human acute myeloid leukemic stem cells, Nat. Med. 12 (2006) 1167-1174.

95. C. Calabrese, H. Poppleton, M. Kocak, T.L. Hogg, C. Fuller, B. Hamner, et al, A perivascular niche for brain tumor stem cells, Cancer Cell 11 (2007) 69-82.

96. M.B. Thomas, J.S. Morris, R. Chadha, M. Iwasaki, H. Kaur, E. Lin, et al, Phase II trial of the combination of bevacizumab and erlotinib in patients who have advanced hepatocellular carcinoma, J. Clin. Oncol. 27 (2009) 843-850.

97. P.N. Kelly, A. Dakic, J.M. Adams, S.L. Nutt, A. Strasser, et al, Tumor growth need not be driven by rare cancer stem cells, Science 317 (2007) 337. 


\section{Figure legends}

Fig. 1 Hepatogenesis and the regulatory machinery involved. In the developing liver, both fibroblast growth factor (FGF) signal from the cardiac mesoderm and bone morphogenetic protein (BMP) signal from the septum transversum mesenchyme cells promote the commitment of ventral endoderm to the liver bud. In the liver bud, hepatic stem cells generate hepatoblasts, bipotent progenitor cells which subsequently give rise to hepatocytes and cholangiocytes.

Fig. 2 Proposed model for the cellular origin of liver malignancies. Liver tumors develop from more heterogeneous cells as expected. Combined hepatocellular and cholangiocellular carcinoma (HC-CC) and hepatoblastoma (HB) are thought to be derived from primitive hepatoblasts and pluripotent stem cells. It remains unclear whether hepatocellular carcinoma (HCC) and cholangiocellular carcinoma (CCC) originate from mature cells or stem/progenitor cells.

Fig. 3 Organization of cancer stem cell systems. Normal stem cells with self-renewal capability generate progenitor cells and subsequently various terminally differentiated cells. Oncogenic mutations in normal stem/progenitor cells or even in differentiated cells enhance or endow the self-renewal capability. Consequently, these cells function as cancer stem cells and contribute to the formation of bulk tumors.

Fig. 4 Hierarchical diversity in cancer cells. Not all types of tumors have a cancer stem cell (CSC) system and CSCs do not necessarily represent a minor subpopulation of cancer cells. Asymmetrical self-renewal division of CSCs is crucial for the maintenance of a hierarchical organization in the tumor. On the contrary, predominant symmetrical self-renewal division of CSCs ultimately produces a highly homogenous 
population in terms of tumorigenicity as shown in melanoma cells [43]. 
Chiba et al. Fig. 1
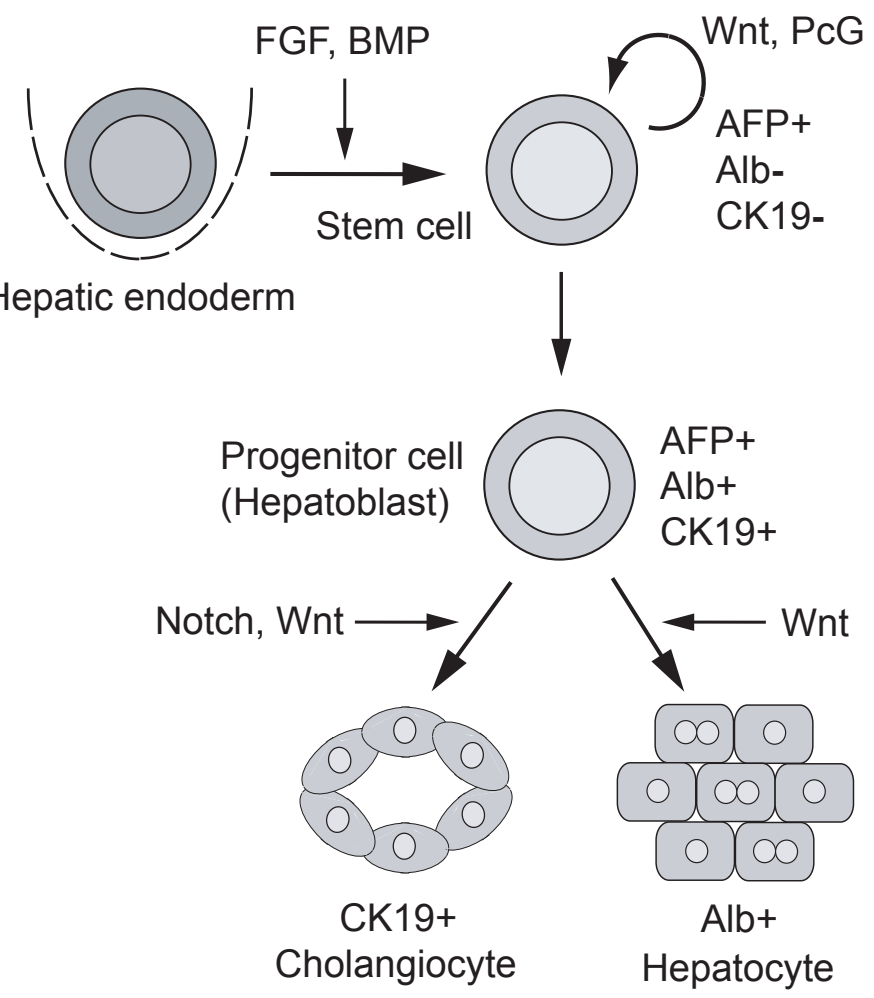
Chiba et al. Fig. 2

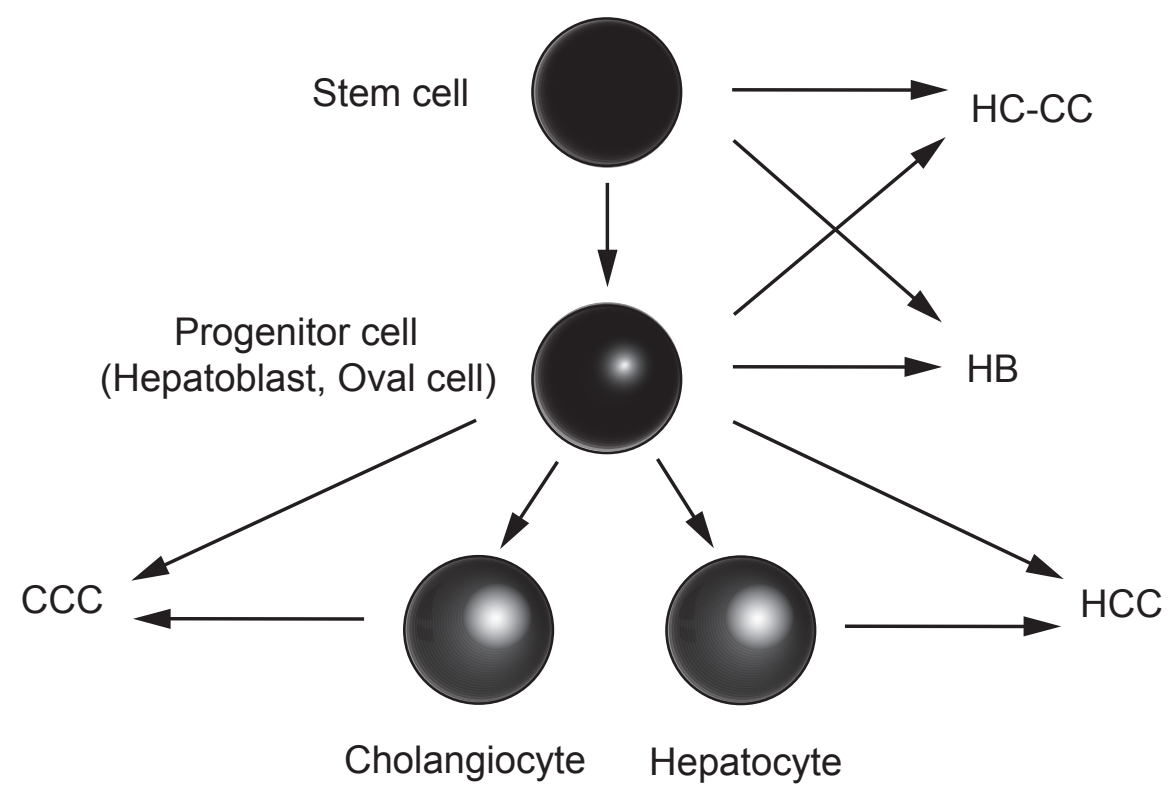


Chiba et al. Fig. 3

Differentiation status

Self-renewal capability

Stem cell Progenitor cell Differentiated cell
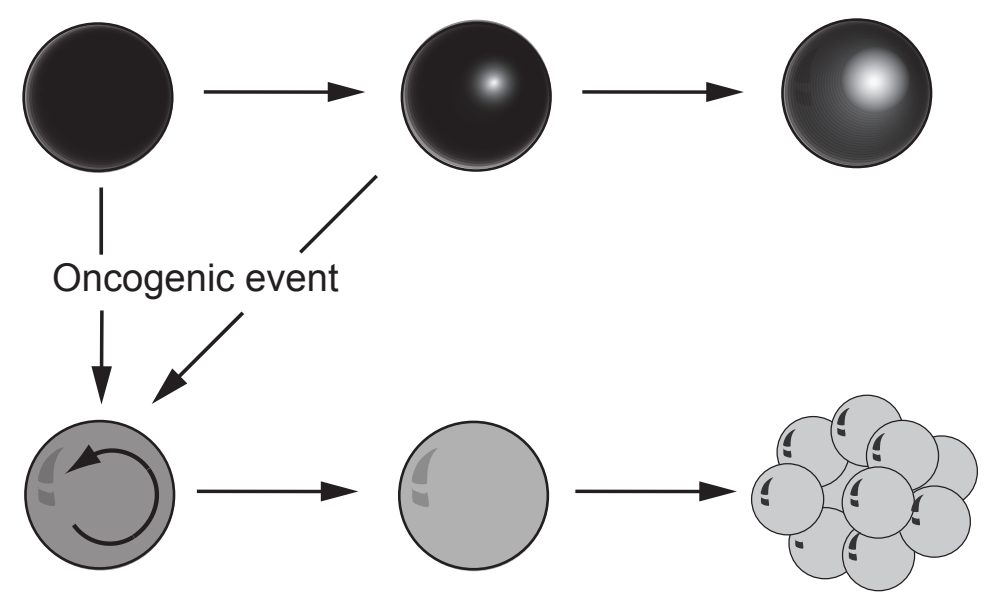

Cancer stem cell

Tumor formation

Tumorigenic potential

Self-renewal capability 
Chiba et al. Fig. 4

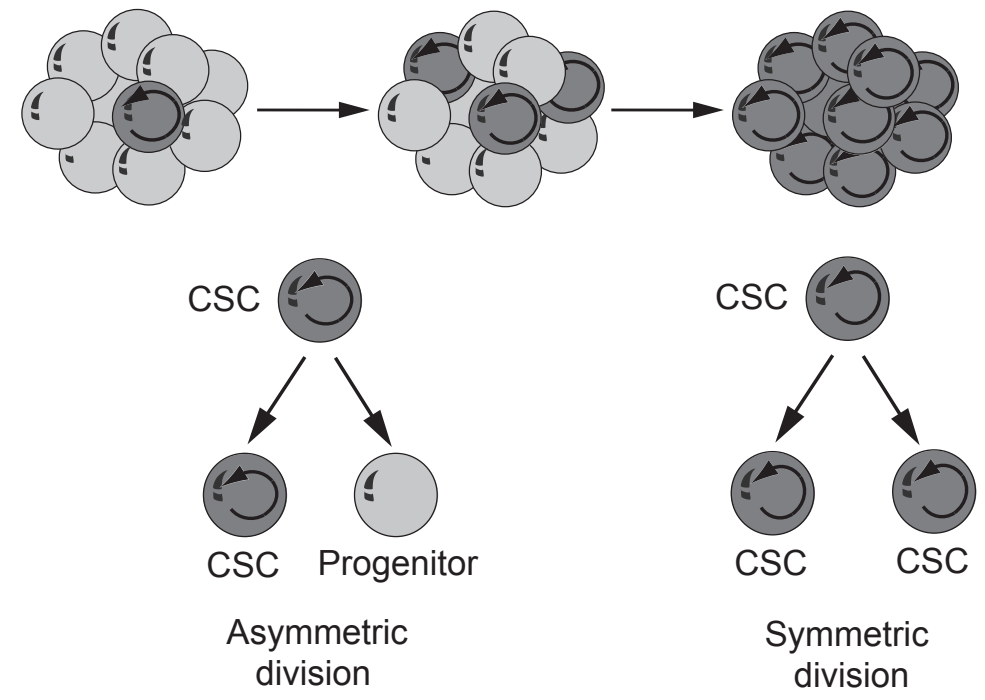


Table1 Cell surface markers for hepatic stem/progenitor cells

\begin{tabular}{|c|c|c|c|c|}
\hline Surface markers & \multicolumn{2}{|l|}{ Source } & Frequency & Reference \\
\hline \multicolumn{5}{|l|}{ Fetal liver } \\
\hline RT1A $1^{-} \mathrm{OX} 18^{\text {low }} \mathrm{ICAM}^{-} 1^{+}$ & \multicolumn{3}{|c|}{ rat (E13) } & {$[16]$} \\
\hline $\operatorname{Liv}^{+}$ & \multicolumn{2}{|c|}{ mouse (ED10.5-12.5) } & $20-60 \%$ & [17] \\
\hline E-cadherin $^{+}$ & \multicolumn{2}{|c|}{ mouse (ED12.5) } & & {$[18]$} \\
\hline Dlk-1 ${ }^{+}$ & \multicolumn{2}{|c|}{ mouse (ED14.5) } & nearly $10 \%$ & [19] \\
\hline Dlk- $1^{+}$ & \multicolumn{2}{|c|}{ rat (ED14) } & $5.7 \pm 0.9 \%$ & {$[20]$} \\
\hline 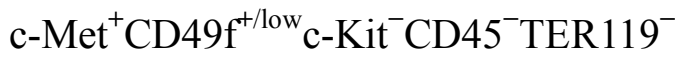 & \multicolumn{2}{|c|}{ mouse (ED13.5) } & $1.90 \pm 0.33 \%$ & {$[21]$} \\
\hline $\mathrm{EPCAM}^{+}$ & \multicolumn{2}{|c|}{ human (16-20 wk fetus) } & $12.1 \pm 2.3 \%$ & {$[22]$} \\
\hline \multicolumn{5}{|l|}{ Adult liver } \\
\hline \multirow[t]{2}{*}{$\mathrm{EpCAM}^{+}$} & \multirow[t]{2}{*}{ human } & (pediatric) & $2.1 \pm 1.6 \%$ & {$[23]$} \\
\hline & & (adult) & $1.3 \pm 1.0 \%$ & \\
\hline \multicolumn{5}{|l|}{ Injured Liver } \\
\hline $\mathrm{CD} 133^{+} \mathrm{CD}^{-} 5^{-}$ & mouse & (DDC diet) & $<0.05 \%$ & {$[25]$} \\
\hline Thy- $1^{+}$ & rat & (AAF/PH or AAF/CCl4) & & {$[26]$} \\
\hline Sca- $1^{+} \mathrm{CD} 34^{+} \mathrm{CD} 45^{+}$ & mouse & (DDC diet) & & {$[27]$} \\
\hline $\mathrm{EpCAM}^{+}$ & rat & $(\mathrm{AAF} / \mathrm{PH})$ & & {$[30]$} \\
\hline
\end{tabular}


Table2 Cell surface markers for CSCs in HCC

\begin{tabular}{|c|c|c|c|}
\hline Surface markers & Frequency (HCC cell lines analyzed) & $\begin{array}{l}\text { Minimal No. of cells } \\
\text { initiating tumors }\end{array}$ & Reference \\
\hline \multicolumn{4}{|l|}{ Cell line } \\
\hline Side population & $0.25-0.80 \%(\mathrm{Huh} 7, \mathrm{PLC} / \mathrm{PRF} / 5)$ & $1 \times 10^{3}$ cells & [35] \\
\hline $\mathrm{CD}_{133^{+}}$ & $0.1-2.0 \%(\mathrm{SMMC} 7721)$ & $1 \times 10^{2}$ cells & [33] \\
\hline $\mathrm{CD} 133^{+}$ & 8-90\% (HepG2, Huh7, PLC8024, Hep3B) & $1 \times 10^{3}$ cells & [37] \\
\hline $\mathrm{OV}^{+}$ & $\begin{array}{l}0.2-3.0 \% \\
\text { (Huh7, SMMC7721, Hep3B, PLC, HepG2) }\end{array}$ & $5 \times 10^{3}$ cells & {$[40]$} \\
\hline $\mathrm{EpCAM}^{+}$ & $58.1-99.2 \%$ (Huh1, Huh7, Hep3B) & $2 \times 10^{2}$ cells & [41] \\
\hline $\mathrm{CD} 0^{+} \mathrm{CD} 44^{+}$ & $\begin{array}{l}0.02-2.53 \% \\
\text { (HepG2, Hep3B, PLC, Huh7, MHCC97L, N }\end{array}$ & $\begin{array}{l}5 \times 10^{2} \text { cells } \\
7 \mathrm{H})\end{array}$ & {$[42]$} \\
\hline \multicolumn{4}{|l|}{ Primary tumor } \\
\hline $\mathrm{CD} 90^{+} \mathrm{CD} 44^{+}$ & $0.74-4.14 \%$ & $2.5 \times 10^{3}$ cells & {$[42]$} \\
\hline
\end{tabular}

